\title{
Inactivated Wnt signaling in resveratrol-treated epidermal squamous cancer cells and its biological implication
}

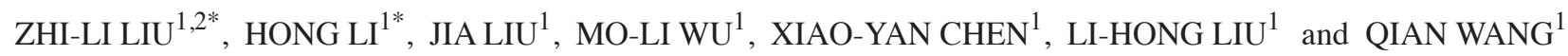 \\ ${ }^{1}$ Liaoning Laboratory of Cancer Genomics and Department of Cell Biology, Dalian Medical University, Dalian, \\ Liaoning 116044; ${ }^{2}$ Department of Dermatology, Dalian Hospital for Skin Diseases, Dalian, Liaoning 116021, P.R. China
}

Received November 6, 2015; Accepted April 13, 2017

DOI: $10.3892 / \mathrm{ol} .2017 .6458$

\begin{abstract}
Squamous cell carcinoma (SCC) is the most common epidermal malignancy, and $\mathrm{Wnt} / \beta$-catenin signaling is frequently activated in SCC. Resveratrol prevents rodent epidermal carcinogenesis, while its effect on human epidermal cancer remains unknown. To address this issue, the impact of resveratrol on the growth and Wnt signaling of skin SCC Colo16 cells were investigated at the cellular and molecular biology levels by flow cytometry, immunocytochemistry, reverse transcription-polymerase chain reaction, western blotting and $\beta$-catenin-specific small interfering RNA (siRNA) transfection. Resveratrol $(100 \mu \mathrm{M})$ suppressed cell growth and induced apoptosis in Colol6 cells. Wnt2 and its downstream genes were downregulated, which was accompanied by increased expression of the Wnt signaling inhibitor Axin2. Transfection with a $\beta$-catenin-specific siRNA did not affect cell growth but enhanced the resveratrol susceptibility of Colo16 transfectants. The present results suggest the inhibitory effects of resveratrol on epidermal SCCs and inactivation of Wnt signaling as one of the resveratrol-caused molecular events in Colo16 cells. $\beta$-catenin oriented siRNA is insufficient to induce cell crisis, implicating the presence of more critical cancer-associated element(s) as the target in Colo16 cells.
\end{abstract}

\section{Introduction}

Squamous cell carcinoma (SCC) is the most common skin malignancy, with aggressive behavior and poor prognosis at the advanced stage. Surgery remains the first choice of SCC treatment, but the highly invasive tendency and chemoresistance to local therapy of SCC cells usually lead to in situ

Correspondence to: Dr Qian Wang, Liaoning Laboratory of Cancer Genomics and Department of Cell Biology, Dalian Medical University, 9 Western Section, Lvshun South Street, Dalian, Liaoning 116044, P.R. China

E-mail: wangqiand173@163.com

*Contributed equally

Key words: resveratrol, epidermal squamous cell carcinoma, Wnt/ $\beta$-catenin signaling, siRNA, apoptosis occurrence and distal dissemination (1). Therefore, exploring a better adjuvant therapy for advanced skin SCCs would improve patients' life quality and survival rates.

Resveratrol possesses a wide range of biological activities, including cancer preventive and therapeutic effects (2). Previous studies performed on rodent models revealed that resveratrol regulated apoptosis and cell survival in mouse skin tumors (3), and exerted chemopreventive effects against ultraviolet-B exposure-mediated damages in SKH-1 hairless mouse skin (4). Nevertheless, the impact of resveratrol on human epidermal SCCs has been less described. The current study therefore aims to investigate i) the biological effects of resveratrol on human epidermal SCC cells; ii) the status of Wnt signaling in resveratrol-treated cells; and iii) the response of normally cultured and resveratrol-treated Colo16 cells when Wnt signal transduction is specifically suppressed by $\beta$-catenin-specific small interfering RNA (siRNA).

\section{Materials and methods}

Cells and treatment. Colo16 human cutaneous SCC cells were cultured in RPMI 1640 medium supplemented with $10 \%$ fetal bovine serum (both Gibco; Thermo Fisher Scientific, Inc., Waltham, MA, USA) at $37^{\circ} \mathrm{C}$ in $5 \% \mathrm{CO}_{2}$ (5). Resveratrol (Sigma-Aldrich; Merck KGaA, Darmstadt, Germany) was dissolved in dimethylsulfoxide (DMSO) and diluted with culture medium to $100 \mu \mathrm{M}$ just prior to use. The treatments lasted for $72 \mathrm{~h}$, and the cells were collected in 24-h intervals for different experimental purposes. Normally cultured cells and cells cultured in medium containing $0.2 \%$ DMSO were used as controls. The experiments were repeated $\geq 3$ times to establish a reliable conclusion.

Cellular and molecular examinations. Hematoxylin and eosin (H\&E) morphological staining and immunocytochemical(ICC) staining for Ki-67 (sc-23900; Santa Cruz, Biotechnology, Inc., Dallas, TX, USA), Wnt2 (sc-514382; Santa Cruz Biotechnology, Inc.), Wnt5a (sc-365376; Santa Cruz Biotechnology, Inc.), $\beta$-catenin (sc-7963; Santa CruzBiotechnology, Inc.), cyclin D1 (sc-8396; Santa Cruz Biotechnology, Inc.), c-Myc (sc-40; Santa Cruz Biotechnology, Inc.), vascular endothelial growth factor (VEGF; sc-7269; Santa Cruz Biotechnology, Inc.), surviving (sc-17779; Santa Cruz, Biotechnology, Inc.) and Axin2 (BS7417; Bioworld Technology, Inc., St Louis Park, 
MN, USA) were performed on cell-bearing coverslips by methods described elsewhere (6,7). The proliferation activity and death pattern of resveratrol-treated Colo16 cells were further analyzed by flow cytometry (BD Biosciences, San Jose, CA, USA) and terminal deoxynucleotidyl transferase dUTP nick-end labeling colorimetric apoptotic cell assay (Promega Corporation, Madison, WI, USA), as previously described (7). The cell viability was determined after treatment for 24 or $48 \mathrm{~h}$ with different concentrations of resveratrol using an MTT assay as previously described (7). The results are presented as the percentage of cell viability [optical density (OD) of the experiment samples/OD of the control] or OD values. The 50\% inhibitory concentration $\left(\mathrm{IC}_{50}\right)$ value was statistically analyzed by SPSS version 15.0 (SPSS, Inc., Chicago, IL, USA). RNA and protein samples were prepared from the experimental groups and subjected to reverse transcription-polymerase chain reaction (RT-PCR) and western blot analyses for the same parameters evaluated by ICC staining (Table I) $(6,8)$.

Transfection of $\beta$-catenin RNA interference (RNAi). Since $\beta$-catenin is the central player of Wnt2-mediated signaling (9), the influence of $\beta$-catenin downregulation in the resveratrol sensitivity of Colo16 cells was evaluated by RNAi transfection according to the manufacturer's protocol (Roche Diagnostics GmbH, Mannheim, Germany). Three RNAi candidate sequences for the $\beta$-catenin transcript were transfected into the cells for $24 \mathrm{~h}$ at a final concentration of $0.5 \mathrm{nmol} / 1$. Scrambled oligonucleotides (mock RNA; sense 5'-UUCUCCGAACGU GUCACGUTT-3' and antisense 5'-ACGUGACACGUUCGG AGA-3') and p53 siRNAs (sense 5'-CUACUUCCUGAAAAC AACGdTdT-3' and antisense 5'-CGUUGUUUUCAGGAA GUAGdTdT-3') were used as negative and positive controls of transfection efficiency, respectively. The siRNAs were synthesized by Shanghai Genepharma, Co., Ltd., Shanghai, China. Upon ascertaining the efficiency of $\beta$-catenin inhibition according to the gray analysis, Colo16 transfectants were further treated with $100 \mu \mathrm{M}$ resveratrol, and the cellular responses were evaluated by H\&E staining, flow cytometry and ICC staining (7).

\section{Results}

Resveratrol inhibits the growth of Colo16 cells. Colo16 SCC cells were treated with $0,50,75,100,125$ and $150 \mu \mathrm{M}$ resveratrol for $72 \mathrm{~h}$. The $\mathrm{IC}_{50}$ value was determined to be $113.79 \mu \mathrm{M}$ (data not shown); therefore, $100 \mu \mathrm{M}$ resveratrol was selected to treat Colo16 SCC cells in subsequent experiments. After $36 \mathrm{~h}$ of treatment, decreased Ki-67 production and apoptotic phenotypes could be observed in the Colo16 cell population, which became particularly distinct at $48 \mathrm{~h}$. The majority of treated cells died at $72 \mathrm{~h}$ (Fig. 1A). Flow cytometry analysis further demonstrated accumulation of S-phase cells and increased apoptotic fraction (16.804\%) in resveratrol-treated cells at $48 \mathrm{~h}$ (Fig. 1B) and MTT assay showed growth suppression (Fig 1C).

Resveratrol inhibits Wnt activation. The expression of Wnt2, Wnt5a and four target genes of the classical Wnt signaling pathway in Colo16 cells with and without resveratrol treatment was examined by RT-PCR and western blot analyses. As shown in Fig. 2, constitutive expression of Wnt2, Wnt5a, survivin,
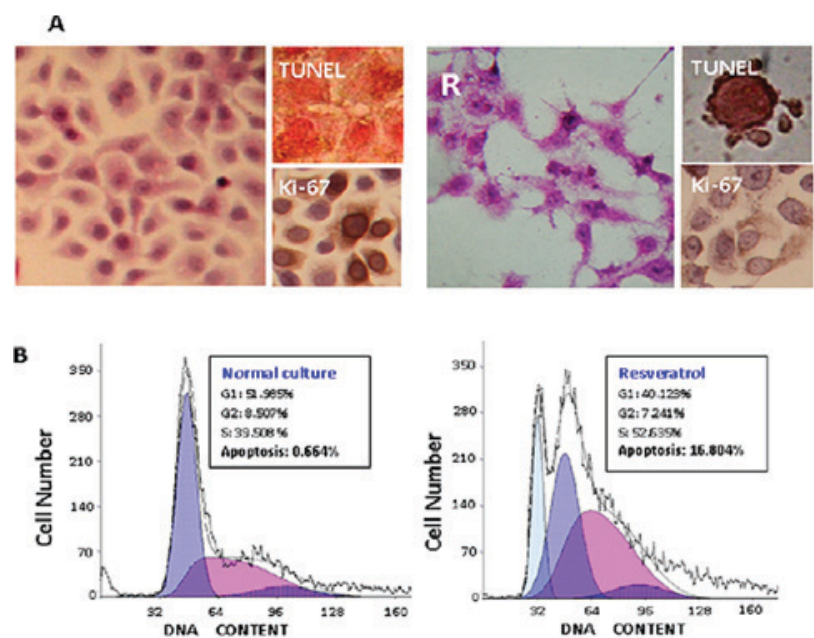

C

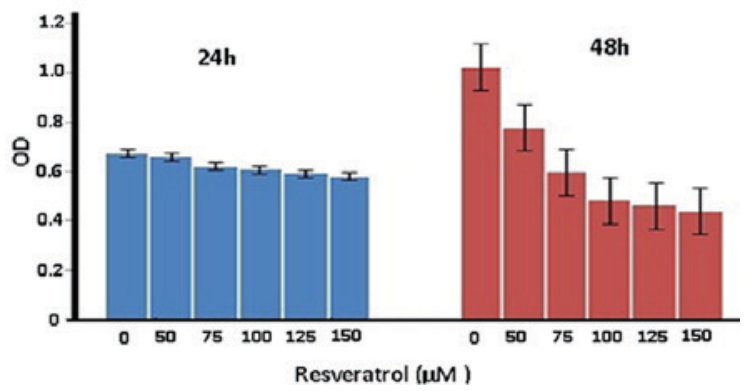

Figure 1. Effects of resveratrol on Colo16 human cutaneous squamous cell carcinoma cells. (A) Evaluation of the response of Colo16 cells to $100 \mu \mathrm{M}$ resveratrol by hematoxylin and eosin morphological staining, immunocytochemical staining for Ki-67 and TUNEL apoptotic cell labeling. (B) Flow cytometry fractionation of cell cycle phases and apoptosis in Colo16 cell populations without and with resveratrol treatment for $48 \mathrm{~h}$. (C) MTT results following treatment for 24 or $48 \mathrm{~h}$ with various concentrations of resveratrol. TUNEL, terminal deoxynucleotidyl transferase dUTP nick-end labeling.

VEGF, cyclin D1 and c-Myc was observed in Colo16 cells under normal culture conditions. Upon resveratrol treatment, Wnt2, survivin, cyclin D1, c-Myc and VEGF transcription was downregulated, while the levels of Wnt5a expression were almost unchanged. As presented in Table II, the expression of Axin2, a negative regulator of the Wnt signaling pathway, was upregulated in resveratrol-treated Colo16 cells (10).

Enhanced resveratrol sensitivity by $\beta$-catenin siRNA transfection. RT-PCR analysis revealed that $\beta$-catenin expression was inhibited by siRNA approach. According to the results of gray analysis, the siRNA candidates in the sequences of sense 5'-GCUUUAUUCUCCCAUUGAATT-3' and antisense 5'-UUCAAUGGGAGAAUAAAGCAG-3' exhibited a marked inhibitory effect (63\%) on $\beta$-catenin expression (Fig. 3B), which was accompanied with less frequent $\beta$-catenin nuclear translocation in the transfectants (Fig. 3A). Distinct apoptosis $(14.896 \%)$ appeared much earlier in resveratrol-treated transfectants than in Colo16 cells treated by resveratrol only (Fig. 3A and C). Almost all cells in the Res + RNAi group died at the 48-h time point.

\section{Discussion}

Resveratrol exerts therapeutic effects on different types of human malignancies at a concentration of $100 \mu \mathrm{M}$ (11-13), 
Table I. The primer sequences for reverse transcription-quantitative polymerase chain reaction.

\begin{tabular}{|c|c|c|c|}
\hline Gene & Primers & $\begin{array}{l}\text { Amplicon } \\
\text { Size (bp) }\end{array}$ & $\begin{array}{c}\text { Annealing } \\
\text { temperature }\left({ }^{\circ} \mathrm{C}\right)\end{array}$ \\
\hline \multirow[t]{2}{*}{ Wnt2 } & F: 5'-GCCACACGCTGCACCTAAAGC-3' & & \\
\hline & R: 5'-CAATTACCCTAAGGGTGGTAGC-3' & 379 & 63 \\
\hline \multirow[t]{2}{*}{ Wnt5a } & F: 5'-CTAACTTAGCTGTGTGGGACATG-3' & & \\
\hline & R: 5'-AAATGCAGAAAGCAAGCTAGCAG-3' & 254 & 60 \\
\hline \multirow[t]{2}{*}{ Axin 2} & F: 5'-GGTGTTTGAGGAGATCTGGG-3' & & \\
\hline & R: 5'-TGCTCACAGCCAAGACAGTT-3' & 153 & 58 \\
\hline \multirow[t]{2}{*}{ Survivin } & F: 5'-GGC ATG GGT GCC CCG ACG TTG-3' & & \\
\hline & R: 5'-CAG AGG CCT CAA TCC ATG GCA-3' & 439 & 58 \\
\hline \multirow[t]{2}{*}{ c-myc } & F: 5'-TGGTCTTCCCCTACCCTCTCAAC-3' & & \\
\hline & R: 5'-GATCCAGACTCTGACCTTTTGCC-3' & 265 & 56 \\
\hline \multirow[t]{2}{*}{ Cyclin D1 } & F: 5'-CTGTGCTGCGAAGTGGAA ACCAT-3' & & \\
\hline & R: 5'-TTCATGGCCAGCGGGAAGACCTC-3' & 257 & 57 \\
\hline \multirow[t]{2}{*}{ VEGF } & F: 5'-CGA AGTGGTGAAGTTCATGGATG-3' & & \\
\hline & R: 5'-TTCTGTATCAGTCTTTCCTGGT-3' & 470 & 60 \\
\hline \multirow[t]{2}{*}{$\beta$-actin } & F: 5'-GCATGGAGTCCTGTGGCAT-3' & & \\
\hline & R: 5'-CATGAAGCATTTGCGGTGG-3' & 326 & 58 \\
\hline
\end{tabular}

Bp, base pair; F, forward; R, reverse; VEGF, vascular endothelial growth factor.

Table II. Resveratrol-regulated gene expression in Colo16 cells.

\begin{tabular}{lccc}
\hline Gene & ICC & Western blotting & RT-PCR \\
\hline Wnt2 & $\downarrow$ & - & $\downarrow$ \\
Wnt5a & - & - & - \\
Axin2 & $\uparrow$ & $\uparrow$ & $\uparrow$ \\
Survivin & $\downarrow$ & $\downarrow$ & $\downarrow$ \\
c-Myc & $\downarrow$ & $\downarrow$ & $\downarrow$ \\
cyclin D1 & $\downarrow$ & $\downarrow$ & $\downarrow$ \\
VEGF & $\downarrow$ & $\downarrow$ & $\downarrow$ \\
\hline
\end{tabular}

ICC, immunocytochemical; RT-PCR, reverse transcription-polymerase chain reaction; VEGF, vascular endothelial growth factor.

while its influence in the growth and survival of human cutaneous SCCs remains obscure. To shed light on this issue, Colo16 cutaneous SCC cells were treated with $100 \mu \mathrm{M}$ resveratrol in the present study. Decreased Ki-67 production and apoptotic phenotypes in the Colo16 cell population were markedly distinct at $48 \mathrm{~h}$, and the majority of treated cells died at $72 \mathrm{~h}$. Accumulation of S-phase cells and increased apoptotic fraction (16.804\%) in resveratrol-treated cells were observed at $48 \mathrm{~h}$. These results suggest that Colo16 is a resveratrol-sensitive human SCC cell line.

Frequent activation of Wnt signaling has been observed in human cutaneous SCC cells (6), but its biological importance in these cells is less known. Therefore, RT-PCR and western blot analyses were performed in the present study to detect the expression of Wnt2, Wnt5a and target genes of the classical Wnt signaling pathway in Colo16 cells. The results suggest that

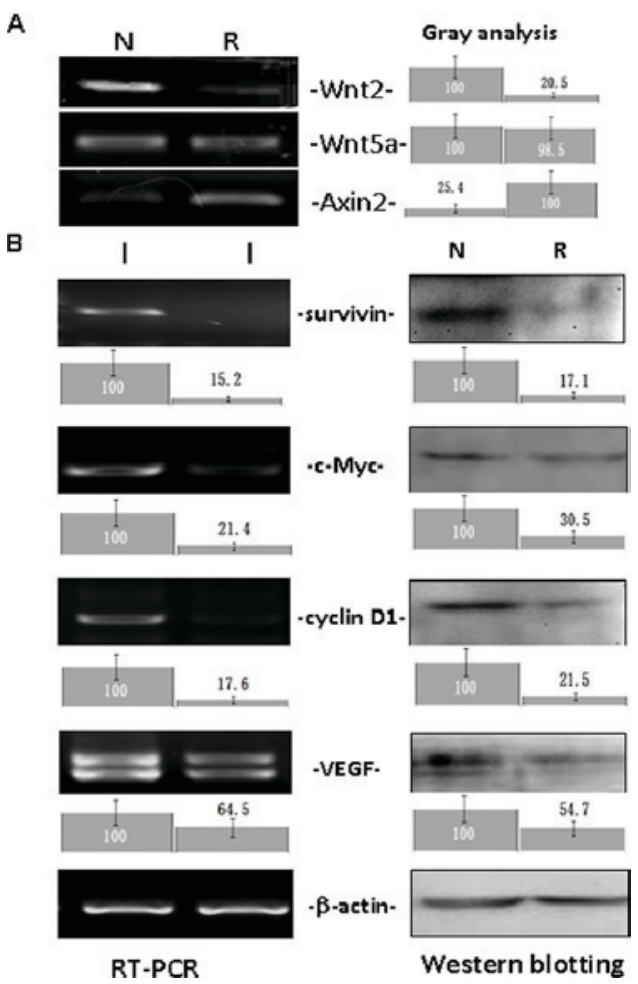

Figure 2. Analyses of Wnt2, Wnt5a, Axin2 and Wnt target gene (survivin, c-Myc, cyclin D1 and VEGF) expression in Colo16 cells without and with $100 \mu \mathrm{M}$ resveratrol treatment for $48 \mathrm{~h}$ by RT-PCR and western blotting. $\beta$-actin was used as a quantitative control. Gray density analysis was conducted on the Wnt2, Wnt5a, Axin2 and Wnt target gene data. N, normal culture; R, $100 \mu \mathrm{M}$ resveratrol treatment; VEGF, vascular endothelial growth factor; RT-PCR, reverse transcription-polymerase chain reaction.

resveratrol can lead Colo16 cells to growth arrest and apoptosis, probably due to inhibition of the classical Wnt signaling 
A

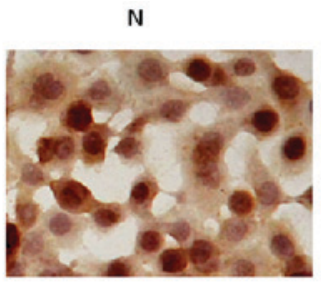

$24 h$ Res

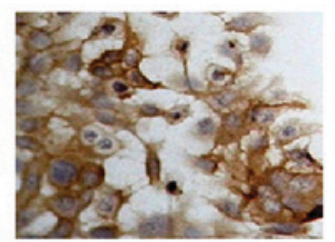

24 h RNAI

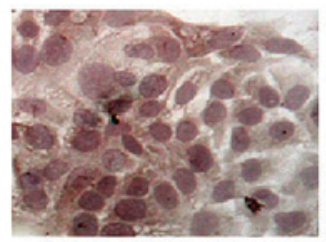

24h RNAi+24h Res

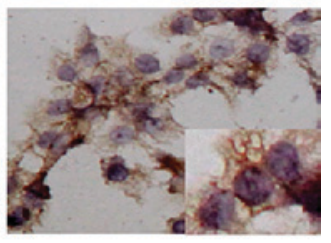

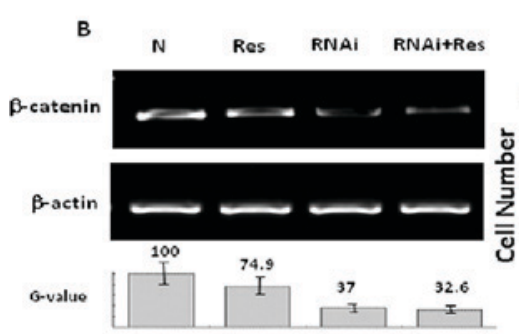

C

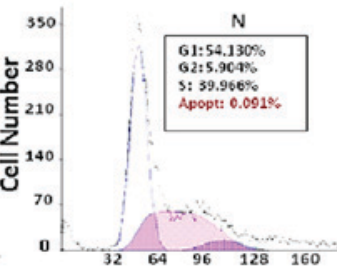

DNA CONTENT

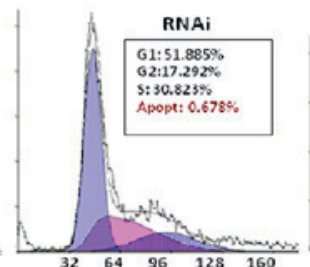

DNA CONTENT

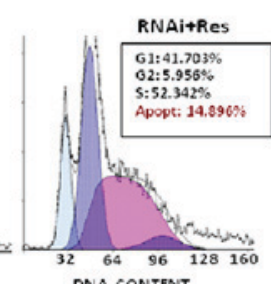

DNA CONTENT

Figure 3. Influence of $\beta$-catenin-specific siRNA in the growth and resveratrol sensitivity of Colo16 cells. (A) $\beta$-catenin immunocytochemical examination of the inhibitory effects of a $\beta$-catenin-specific siRNA and its combination with 24-h treatment of $100 \mu \mathrm{M}$ resveratrol on the $\beta$-catenin expression of Colo16 cells. Normally cultured cells were used as controls. (B) Reverse transcription-polymerase chain reaction and gray density analysis of $\beta$-catenin expression in Colol6 cells under normal culture conditions, or treated with $100 \mu \mathrm{M}$ resveratrol for $48 \mathrm{~h}$, RNAi or RNAi and $100 \mu \mathrm{M}$ resveratrol for $24 \mathrm{~h}$. (C) Flow cytometry was performed on $\beta$-catenin siRNA transfectants without RNAi and with $100 \mu \mathrm{M}$ resveratrol treatment at $24 \mathrm{~h}$. Colo16 cells treated with $100 \mu \mathrm{M}$ resveratrol only were used as the control. N, normal culture; Res, resveratrol; RNAi, RNA interference; siRNA, small interfering RNA.

pathway and downregulation of certain Wnt target genes that are critical for human SCCs $(6,14,15)$. This notion was further supported by the remarkable upregulation of Axin2 expression in resveratrol-treated Colo16 cells, since Axin2 acts as a negative regulator of the Wnt signaling pathway by promoting glycogen synthase kinase $3 \beta$-dependent phosphorylation of $\beta$-catenin (16). The unchanged Wnt5a expression in resveratrol-treated Colo16 cells observed in the present study was not unexpected, as Wnt5a mediates non-canonical (i.e. $\beta$-catenin-independent) signaling and functions as a negative regulator of $\mathrm{Wnt} / \beta$-catenin activity (17) or as a tumor-suppressor gene (18).

$\beta$-catenin serves a central role in the classical Wnt signaling pathway through nuclear translocation to initiate Wnt target gene transcription (9). To ascertain the importance of Wnt activation in Colo16 cells, $\beta$-catenin expression was inhibited by siRNA approach in the present study. The results revealed that $\beta$-catenin siRNA treatment by itself failed to cause cell death, suggesting that the suppressed biological activities of Wnt signaling may be compensated by other activated signaling pathways to maintain the survival of Colo16 cells. Furthermore, distinct apoptosis (14.896\%) appeared much earlier in resveratrol-treated transfectants than in Colo16 cells treated with resveratrol only. This phenomenon further suggested multiple molecular targeting features of resveratrol in cancer cells $(13,19)$. In this context, resveratrol treatment rather than a signaling-specific strategy may achieve better therapeutic effects on human epidermal SCCs.

\section{Acknowledgements}

The present study was supported by grants from the National Natural Science Foundation of China (grant nos. 81450016, 81272786, 81071971, 81072063 and 30971038), Research Fund for PhD supervisors from the National Education Department of China (grant no. 20122105110005), Program
Fund for Liaoning Excellent Talents in University (grant no. LJQ2012078) and Program Fund for Liaoning Natural Science Foundation (grant nos. 2013023050 and 2013023040).

\section{References}

1. Kim RH and Armstrong AW: Nonmelanoma skin cancer. Dermatol Clin 30: 125-139, ix, 2012

2. Singh CK, Ndiaye MA and Ahmad N: Resveratrol and cancer: Challenges for clinical translation. Biochim Biophys Acta 1852: 1178-1185, 2015.

3. George J, Singh M, Srivastava AK, Bhui K, Roy P, Chaturvedi PK and Shukla Y: Resveratrol and black tea polyphenol combination synergistically suppress mouse skin tumors growth by inhibition of activated MAPKs and p53. PLoS One 6: e23395, 2011.

4. Aziz MH, Reagan-Shaw S, Wu J, Longley BJ and Ahmad N: Chemoprevention of skin cancer by grape constituent resveratrol: Relevance to human disease? FASEB J 19: 1193-1195, 2005.

5. Moore GE, Merrick SB, Woods LK and Arabasz NM: A human squamous cell carcinoma cell line. Cancer Res 35: 2684-2688, 1975.

6. Li Y, Liu ZL, Zhang KL, Chen XY, Kong QY, Wu ML, Sun Y, Liu J and Li H: Methylation-associated silencing of S100A4 expression in human epidermal cancers. Exp Dermatol 18: 842-848, 2009

7. Xia SL, Wu ML, Li H, Wang JH, Chen NN, Chen XY, Kong QY, Sun $\mathrm{Z}$ and Liu J: CRABP-II- and FABP5-independent responsiveness of human glioblastoma cells to all-trans retinoic acid. Oncotarget 6: 5889-5902, 2015.

8. Liu ZL, Li Y, Kong QY, Zhan C, Wang Q, Chen XY, Sun Y, Wen S, Tu CX, Liu J and Li H: Immunohistochemical profiling of Wnt, NF-kappaB, Stat3 and Notch signaling in human epidermal tumors. J Dermatol Sci 52: 133-136, 2008.

9. Skalka N, Caspi M, Caspi E, Loh YP and Rosin-Arbesfeld R: Carboxypeptidase E: A negative regulator of the canonical Wnt signaling pathway. Oncogene 32: 2836-2847, 2013.

10. Wu ZQ, Brabletz T, Fearon E, Willis AL, Hu CY, Li XY and Weiss SJ: Canonical Wnt suppressor, Axin2, promotes colon carcinoma oncogenic activity. Proc Natl Acad Sci USA 109: 11312-11317, 2012.

11. Mikuła-Pietrasik J, Sosińska P and Książek K: Resveratrol inhibits ovarian cancer cell adhesion to peritoneal mesothelium in vitro by modulating the production of $\alpha 5 \beta 1$ integrins and hyaluronic acid. Gynecol Oncol 134: 624-630, 2014.

12. Wang Q, Li H, Wang XW, Wu DC, Chen XY and Liu J: Resveratrol promotes differentiation and induces Fas-independent apoptosis of human medulloblastoma cells. Neurosci Lett 351: 83-86, 2003. 
13. Wen S, Li H, Wu ML, Fan SH, Wang Q, Shu XH, Kong QY, Chen $\mathrm{XY}$ and Liu J: Inhibition of $\mathrm{NF}-\mathrm{\kappa B}$ signaling commits resveratrol-treated medulloblastoma cells to apoptosis without neuronal differentiation. J Neurooncol 104: 169-177, 2011.

14. Klaus A and Birchmeier W: Wnt signalling and its impact on development and cancer. Nat Rev Cancer 8: 387-398, 2008.

15. Malanchi I, Peinado H, Kassen D, Hussenet T, Metzger D, Chambon P, Huber M, Hohl D, Cano A, Birchmeier W and Huelsken J: Cutaneous cancer stem cell maintenance is dependent on beta-catenin signalling. Nature 452: 650-653, 2008.

16. Bernkopf DB,Hadjihannas MV and Behrens J: Negative-feedback regulation of the Wnt pathway by conductin/axin 2 involves insensitivity to upstream signalling. J Cell Sci 128: 33-39, 2015.
17. Bisson JA, Mills B, Paul Helt JC, Zwaka TP and Cohen ED: Wnt5a and Wnt11 inhibit the canonical Wnt pathway and promote cardiac progenitor development via the Caspase-dependent degradation of AKT. Dev Biol 398: 80-96, 2015.

18. Abdelmaksoud-Dammak R, Miladi-Abdennadher I, Saadallah-Kallel A, Khabir A, Sellami-Boudawara T, Frikha M, Daoud J and Mokdad-Gargouri R: Downregulation of WIF-1 and Wnt5a in patients with colorectal carcinoma: Clinical significance. Tumour Biol 35: 7975-7982, 2014.

19. Yu LJ, Wu ML, Li H, Chen XY, Wang Q, Sun Y, Kong QY and Liu J: Inhibition of STAT3 expression and signaling in resveratrol-differentiated medulloblastoma cells. Neoplasia 10: 736-744, 2008 